VOL. 47 (1993) [509-518]

\title{
TOPOLOGICAL AND ORDER-TOPOLOGICAL ORTHOMODULAR LATTICES
}

\author{
Zdenka Riečanová
}

\begin{abstract}
The necessary and sufficient conditions for atomic orthomodular lattices to have the MacNeille completion modular, or (o)-continuous or order topological, orthomodular lattices are proved. Moreover we show that if in an orthomodular lattice the (o)-convergence of filters is topological then the (o)-convergence of nets need not be topological. Finally we show that even in the case when the MacNeille completion $\tilde{L}$ of an orthomodular lattice $L$ is order-topological, then in general the (o)-convergence of nets in $\widetilde{L}$ does not imply their (o)-convergence in $L$. (This disproves, also for the orthomodular and order-topological case, one statement in G.Birkhoff's book.)
\end{abstract}

\section{INTRODUCTION}

In [17] and [12] compact orthomodular lattices have been studied. In [16] some results from [17] are generalised to locally compact orthomodular lattices. In this paper we generalise some results from [12] to topological and order topological orthomodular lattices.

A well-known fact is that the MacNeille completion of an orthomodular lattice $L$ need not be orthomodular, even if $L$ is a modular ortholattice ([1, 5] and others). Little is known about completions of orthomodular lattices (abbreviated OMLs). Positive results are given by Janovitz in [6] for indexed OMLs; in [3] Brums-Greecie-HardingRoddy showed that a variety generated by a single finite OML is closed under MacNeille completions; in [12] and [14] OMLs have been characterised the MacNeille completions of which are compact topological OMLs or profinite OMLs (see also [17]). For atomic OMLs some positive results are given in part 3 of this article. Part 4 shows that the order-topological OMLs in the language of filters and in the language of nets are distinct.

Received 5th December, 1992.

This research was supported by the grant from the Slovak Academy of Sciences GA-SAV 368/91.

Copyright Clearance Centre, Inc. Serial-fee code: 0004-9729/93 \$A2.00+0.00. 


\section{Basic Definitions AND Lemmas}

By an orthomodular lattice $(L, \vee, \wedge, \perp, 0,1)$ (abbreviated OML) we mean a lattice $(L, \vee, \wedge)$ with 0 and $1(0 \neq 1)$ and with an orthocomplementation $\perp: L \rightarrow L$ such that

(i) $a \leqslant b \Rightarrow b^{\perp} \leqslant a^{\perp}$,

(i) $\left(a^{\perp}\right)^{\perp}=a$,

(iii) $a \vee a^{\perp}=1$,

(iv) $a \leqslant b \Rightarrow b=a \vee\left(a^{\perp} \wedge b\right)$ (the orthomodular law).

We say that two elements $x, y \in L$ are orthogonal if $x \leqslant y^{\perp}$. A set $M \subset L$ is called an orthogonal set of elements if for every $x, y \in L, x \neq y$, we have $x \leqslant y^{\perp}$. A net $\left(x_{\alpha}\right)_{\alpha \in \mathcal{E}} \subset L(0)$-converges to an element $x \in L$ if there are nets $\left(u_{\alpha}\right)_{\alpha \in \mathcal{E}},\left(v_{\alpha}\right)_{\alpha \in \mathcal{E}}$ in $L$ such that $u_{\alpha} \leqslant x_{\alpha} \leqslant v_{\alpha}$ for every $\alpha \in \mathcal{E}$ and $u_{\alpha} \nearrow x, v_{\alpha} \searrow x$ (where $u_{\alpha} \nearrow x$ means that the net $\left(u_{\alpha}\right)_{\alpha \in \mathcal{E}}$ is nondecreasing and $\bigvee_{\alpha} u_{\alpha}=x$; the meaning of $v_{\alpha} \searrow x$ is dual). The order topology $\tau_{0}$ is the strongest (finest) topology on $L$ such that the (o)-convergence of nets implies their topological convergence.

Recall that $a \in L$ is an atom in $L$ if $a \neq 0$ and $(b \leqslant a \Rightarrow b=0$ or $b=a)$. An OML $L$ is atomic if every nonzero element in $L$ contains an atom. In fact an atomic OML is atomistic in the sense that every nonzero element in $L$ is the supremum of all atoms lying under it. Moreover every nonzero element of an atomic OML is a join (supremum) of an orthogonal set of atoms (see [7, p.140]). An element $x \in L$ is called a finite element if $x$ is a join of a finite set of atoms. We use the symbol $\bigvee S$ for the supremum of the set $S \subset L$. An OML $L$ is called (o)-continuous if for any net $\left(x_{\alpha}\right)_{\alpha \in \mathcal{E}} \subset L$ and any $x, y \in L, x_{\alpha} \nearrow x \in L$ implies $y \wedge x_{\alpha} \nearrow y \wedge x$. If in the definition of OML we omit the condition (iv) (orthomodular law) then $L$ is called an ortholattice. The notions of atomic, atomistic and (o)-continuous ortholattice are defined as for OMLs. (Note that an atomic ortholattice need not be atomistic). If $L$ is an (o)-continuous ortholattice then for any $x_{\alpha}, y_{\alpha}, x, y \in L$, we have: $x_{\alpha} \stackrel{(0)}{\longrightarrow x}, y_{\alpha} \stackrel{(0)}{\longrightarrow} y$ implies $x_{\alpha} \vee y_{\alpha} \stackrel{(o)}{\longrightarrow} x \vee y$, $x_{\alpha} \wedge y_{\alpha} \stackrel{(o)}{\longrightarrow} x \wedge y$. For the other terminologies and definitions we refer to [7].

Let $L$ be an atomistic ortholattice and let $A=\{a \in L \mid a$ is an atom $\}$. For every $a \in A$ we define real functions $f_{a}: L \rightarrow\{0,1\}, f_{a \perp}: L \rightarrow\{0,1\}$ as follows

$$
\begin{gathered}
f_{a}(x)=\left\{\begin{array}{ll}
1 & \text { if } a \leqslant x \\
0 & \text { if } a \neq x
\end{array} \quad x \in L,\right. \\
f_{a}(x)=\left\{\begin{array}{ll}
1 & \text { if } x \leqslant a^{\perp} \\
0 & \text { if } x \neq a^{\perp}
\end{array} \quad x \in L .\right.
\end{gathered}
$$


The family of functions $\psi=\left\{f_{a} \mid a \in A\right\} \cup\left\{f_{a} \perp \mid a \in A\right\}$ induces the uniformity $\mathcal{U}_{\psi}$ and the topology $\tau_{\psi}$ (see [4, p.168]); clearly for any net $\left(x_{\alpha}\right)_{\alpha \in \mathcal{E}} \subset L$ and any $x \in L$

$$
x_{\alpha} \stackrel{\tau_{\psi}}{\longrightarrow} x \text { if and only if } \forall a \in A: f_{a}\left(x_{\alpha}\right) \rightarrow f_{a}(x) \text { and } f_{a^{\perp}}\left(x_{\alpha}\right) \rightarrow f_{a^{\perp}}(x)
$$

This implies, in view of the atomisticity of $L$, that the topology $\tau_{\psi}$ is Hausdorff. (For the properties of the topology $\tau_{\psi}$ in OMLs, see $[12,13]$.)

In view of this observation the folloving lemmas can be proved.

Lemma 2.1. Let $L$ be an atomistic ortholattice. Then $\tau_{0} \subset \tau_{\psi}$.

Proof: Since the functions $f_{a}, f_{a}$ are continuous in $\tau_{\psi}$ we find that for every atom $a \in L$ the intervals $[a, 1]=f_{a}{ }^{-1}(\{1\})$ and $\left[0, a^{\perp}\right]=f_{a \perp}{ }^{-1}(\{1\})$ are clopen sets in $\tau_{\psi}$. Let $x \in L, x \neq 0, x \neq 1$. Let $\mathcal{U}(x) \in \tau_{0}$ be such that $x \in \mathcal{U}(x)$. Since $L$ is atomistic we have $x=\bigvee A_{x}, x^{\perp}=\bigvee A_{x \perp}$, where $A_{x}=\{a \in L \mid a \leqslant x, a$ is an atom $\}$, $A_{x^{\perp}}=\left\{a \in L \mid a \leqslant x^{\perp}, a\right.$ is an atom $\}$. Put $C=\left\{\gamma \subset A_{x} \cup A_{x^{\perp}} \mid \gamma \cap A_{x} \neq \emptyset \neq \gamma \cap A_{x^{\perp}}\right.$, $\gamma$ is a finite set\}. Evidently $C$ is directed by the set inclusion. For every $\gamma \in C$ put $x_{\gamma}=\bigvee\left\{a \in L \mid a \in \gamma \cap A_{z}\right\}, y_{\gamma}=\bigwedge\left\{a^{\perp} \in L \mid a \in \gamma \cap A_{z^{\perp}}\right\}$. Then $x_{\gamma} \nearrow x$, $y_{\gamma} \searrow x$ and $\left[x_{\gamma}, y_{\gamma}\right]$ are clopen sets in $\tau_{\psi}$. Suppose that for every $\gamma \in C$ there is $z_{\gamma} \in\left[x_{\gamma}, y_{\gamma}\right]$ such that $z_{\gamma} \notin U(x)$. Since $x_{\gamma} \leqslant z_{\gamma} \leqslant y_{\gamma}$ for every $\gamma \in C$, we get $z_{\gamma} \stackrel{(0)}{\longrightarrow} x$. As $L \backslash U(x)$ is closed in $\tau_{0}$ we find that $x \in L \backslash U(x)$, a contradiction. Thus there exists $\gamma_{0} \in C$ such that $x \in\left[x_{\gamma_{0}}, y_{\gamma_{0}}\right] \subset U(x)$. In a similar way we find that for $x=1$ there exists $\gamma \in C$ such that $\left[x_{\gamma}, 1\right] \subset U(1) \in \tau_{0}$ and for $x=0$ there exists $\gamma \in C$ such that $\left[0, y_{\gamma}\right] \subset U(0) \in \tau_{0}$. We conclude that $\tau_{0} \subset \tau_{\psi}$.

An atomistic ortholattice $L$ we call compactly atomistic if for every $x \in L$ and any set $S$ of atoms in $L$ such that $x=\bigvee S$, the following holds: to every atom $a \leqslant x$ there exists a finite set $\left\{p_{1}, p_{2}, \ldots, p_{k}\right\} \subset S$ such that $a \leqslant \bigvee_{k=1}^{n} p_{k}$. An atomistic ortholattice is called strongly compactly atomistic if to every set $S, S \subset A=\{a \in L \mid a$ is an atom $\}$ and to every $p \in \bar{S}=\left\{p \in A \mid\right.$ if $a \in A$ and $a \leqslant b^{\perp}$ for every $b \in S$ then $\left.p \leqslant a^{\perp}\right\}$ there exists a finite set $\left\{p_{1}, p_{2}, \ldots, p_{k}\right\} \subset S$ such that $p \leqslant \bigvee_{k=1}^{n} p_{k}$. Clearly a complete atomistic ortholattice $L$ is strongly compactly atomistic if and only if $L$ is compactly atomistic.

Lemma 2.2. Let $L$ be an atomistic ortholattice and $A=\{a \in L \mid a$ is an atom $\}$. The following conditions are equivalent:

(a) $L$ is (o)-continuous; 
(b) for every $a \in A$ and any $x_{\alpha}, x \in L\left(\alpha \in \mathcal{E}, \mathcal{E}\right.$ is direcet) $x_{\alpha} \nearrow x$ implies $a \wedge x_{\alpha}>a \wedge x$;

(c) $L$ is compactly atomistic;

(d) $x_{\alpha} \stackrel{(o)}{\longrightarrow x}$ implies $x_{\alpha} \stackrel{\tau_{\psi}}{\longrightarrow} x$, for any $x_{\alpha}, x \in L(\alpha \in \mathcal{E}, \mathcal{E}$ is directed);

(e) $\tau_{\psi}=\tau_{0}$.

Proof: $((a) \Rightarrow(b))$ This is obvious.

$((b) \Rightarrow(c))$ Let $S \subset A$ be such that $\bigvee S=x \in L$. Let a set $\mathcal{E}=\{\alpha \subset S \mid \alpha$ is a finite set\} be directed by the set inclusion. Denote $x_{\alpha}=\bigvee \alpha$ for every $\alpha \in \mathcal{E}$. Then $x_{\alpha} \nearrow x$ and hence $a \in A, a \leqslant x$ implies that there exists $\alpha_{0} \in \mathcal{E}$ such that $a \leqslant x_{\alpha_{0}}=\bigvee \alpha_{0}$

$((c) \Rightarrow(a))$ Let $x_{\alpha}, x, y \in L,\left(\alpha \in \mathcal{E}, \mathcal{E}\right.$ is directed) be such that $x_{\alpha} \nearrow x$. Let $b \in A, b \leqslant x \wedge y$. Since $x=\bigvee\left\{a \in A \mid a \leqslant x_{\alpha}, \alpha \in \mathcal{E}\right\}$, there exists a finite set $\left\{a_{1}, a_{2}, \ldots, a_{n}\right\} \subset\left\{a \in A \mid a \leqslant x_{\alpha}, \alpha \in \mathcal{E}\right\}$ such that $b \leqslant \bigvee_{k=1}^{n} a_{k}$. Suppose $a_{k} \leqslant x_{\alpha_{k}}$ $(k=1,2, \ldots, n)$ and $\alpha_{0} \geqslant \alpha_{k}, k=1,2, \ldots, n$. Then $b \leqslant \bigvee_{k=1}^{n} a_{k} \leqslant \bigvee_{k=1}^{n} x_{\alpha_{k}} \leqslant x_{\alpha_{0}}$ and thus $b \leqslant x_{\alpha_{0}} \wedge y$. We obtain $\bigvee\left\{x_{\alpha} \wedge y \mid \alpha \in \mathcal{E}\right\}=x \wedge y$.

$((d) \Rightarrow(e))$ Clearly $(d)$ implies that $\tau_{\psi} \subset \tau_{0}$, in view of the definition of $\tau_{0}$. Moreover we have $\tau_{0} \subset \tau_{\psi}$ and hence $\tau_{0}=\tau_{\psi}$.

$((e) \Rightarrow(b))$ Suppose $x_{\alpha}, x \in L\left(\alpha \in \mathcal{E}, \mathcal{E}\right.$ is directed) and $x_{\alpha} / x$. Then $x_{\alpha} \stackrel{\tau_{0}}{\rightarrow} x$; hence $x_{\alpha} \stackrel{T_{\psi}}{\longrightarrow} x$. Thus for every $a \in A$ we have $f_{a}\left(x_{\alpha}\right) \rightarrow f_{a}(x)$ and hence $x_{\alpha} \wedge a \nearrow x \wedge a$.

$((b) \Rightarrow(d))$ Since $(b) \Rightarrow(a)$ we see that for every $x_{\alpha}, x \in L(\alpha \in \mathcal{E}, \mathcal{E}$ is directed) such that $x_{\alpha} \stackrel{(0)}{\longrightarrow} x$ and every $a \in A$, it follows that $x_{\alpha} \wedge a \stackrel{(0)}{\longrightarrow} x \wedge a$ and hence $f_{a}\left(x_{\alpha}\right) \rightarrow f_{a}(x)$. Moreover $x_{\alpha}^{\perp} \stackrel{(0)}{\longrightarrow} x^{\perp}$ and this implies $f_{a}\left(x_{\alpha}^{\perp}\right) \rightarrow f_{a}\left(x^{\perp}\right)$ or equivalently $f_{a \perp}\left(x_{\alpha}\right) \rightarrow f_{a \perp}(x)$ (since $f_{\alpha}\left(x^{\perp}\right)=f_{\alpha \perp}(x)$ for every $x \in L$ and $a \in A$ ). We obtain $x_{\alpha} \stackrel{\tau_{\psi}}{\longrightarrow} x$.

LEMma 2.3. Let $L$ be an (o)-continuous, complete and atomistic ortholattice. Then for any net $\left(x_{\alpha}\right)_{\alpha \in \mathcal{E}} \subset L$ and any $x \in L$,

$$
x_{\alpha} \stackrel{\tau_{\psi}}{\longrightarrow} x \text { implies } \quad x_{\alpha} \stackrel{(o)}{\longrightarrow} x .
$$

Proof: Suppose $x_{\alpha}, x \in L\left(\alpha \in \mathcal{E}, \mathcal{E}\right.$ is directed) and $x_{\alpha} \stackrel{\tau_{\psi}}{\longrightarrow} x$. This implies that for every atom $a \in L, a \leqslant x$ there exists $\beta_{a} \in \mathcal{E}$ such that for every $\alpha \geqslant \beta_{a}$, we have $a \leqslant x_{\alpha}$. Thus for every atom $a \leqslant x$ we have $a \leqslant \bigvee_{\beta} \bigwedge_{\alpha \geqslant \beta} x_{\alpha}$ and hence $x \leqslant$ 
$\bigvee_{\beta} \bigwedge_{\alpha \geqslant \beta} x_{\alpha}$. Since $x_{\alpha} \stackrel{\tau_{\phi}}{\longrightarrow} x$ implies $x_{\alpha}^{\perp} \stackrel{\tau_{\phi}}{\longrightarrow} x^{\perp}$ we have also $x^{\perp} \leqslant \bigvee_{\beta} \bigwedge_{\alpha \geqslant \beta} x_{\alpha}^{\perp}$, and hence $x \geqslant \bigwedge_{\beta} \bigvee_{\alpha \geqslant \beta} x_{\alpha}$. Now $x \leqslant \bigvee_{\beta} \bigwedge_{\alpha \geqslant \beta} x_{\alpha} \leqslant \bigwedge_{\beta} \bigvee_{\alpha \geqslant \beta} x_{\alpha} \leqslant x$. This means that $x_{\alpha} \stackrel{(o)}{\longrightarrow} x$.

\section{Topological and Order-topological OMls in the language of Nets}

Examples of atomistic ortholattices are atomic OMLs. Thus using the previous three lemmas we can find: $(a)$ : necessary and sufficient condition under which the MacNeille completion of an (o)-continuous atomic OML is an (o)-continuous OML; (b): a necessary and sufficient condition under which the MacNeille completion of a modular atomic ortholattice is a modular OML. For details about these questions and counterexamples showing that generally this is not fulfilled, see Kalmbach's book [7, p.259].

An atomisitic ortholattice $L$ is called topological if there exists a Hausdorff topology $\tau$ on $L$ such that $x_{\alpha} \stackrel{\tau}{\rightarrow} x, y_{\alpha} \stackrel{\tau}{\rightarrow} y$ implies $x_{\alpha}^{\perp} \stackrel{\tau}{\rightarrow} x^{\perp}, x_{\alpha} \vee y_{\alpha} \stackrel{\tau}{\rightarrow} x \vee y$ and $x_{\alpha} \wedge y_{\alpha} \stackrel{\tau}{\rightarrow} x \wedge y$ for any $x_{\alpha}, y_{\alpha}, x, y \in L$.

A topological ortholattice $L$ (precisely $\left.\left(L, \tau_{0}\right)\right)$ is called order-topological if $L$ is topological for the order topology $\tau_{0}$ and $x_{\alpha} \stackrel{\tau_{0}}{\longrightarrow} x$ implies $x_{\alpha} \stackrel{(0)}{\longrightarrow}$ for any $x_{\alpha}, x \in L$.

THEOREM 3.1. Let $L$ be a complete and atomistic ortholattice. The followng conditions are equivlent:

(i) $L$ is order topological;

(ii) $L$ is (o)-continuous;

(iii) $x_{\alpha} \stackrel{(o)}{\longrightarrow} x$ if and only if $x_{\alpha} \stackrel{\tau_{\psi}}{\longrightarrow} x$ for any net $\left(x_{\alpha}\right)_{\alpha \in \mathcal{E}} \subset L$ and any $x \in L$;

(iv) $L$ is compactly atomistic.

Proof: ((i) $\Rightarrow$ (ii)) This is obvious.

((ii) $\Rightarrow$ (iii)) By Lemmas 2.2 and 2.3 .

((iii) $\Rightarrow$ (i)) In view of Lemma 2.2, (iii) implies that $\tau_{\psi}=\tau_{0}$ and $L$ is (o)-continuous. Hence $L$ is order-topological.

((iv) $\Leftrightarrow$ (i) By Lemma 2.2 and the fact that ((ii) $\Leftrightarrow$ (i)).

Kaplansky [8] has shown that every complete modular ortholattice is (o)-continuous. Using this result we obtain the following corollary of Theorem 3.1.

Corollary 3.2. Every complete atomic modular OML is order-topological.

An example of an order-topological OML which need not be modular is any OML isomorphic to a direct product of finite OMLs. Another example is any complete atomic OML $L$ such that to every atom there exist only finitely many non-orthogonal atoms. (See $[12,17]$.) 
A partially ordered set $P$ can be embedded into a complete lattice $\widetilde{L}$ such that every element $x \in \widetilde{L}$ is a supremum of some subset of $\varphi(P)$ and also an infimum of some subset of $\varphi(P)$ ( $\varphi: P \rightarrow \widetilde{L}$ is the embedding). We say that $P$ is supremum and infimum densely embedded in $\tilde{L}$. It has been shown in [15] that any complete lattice into which $P$ can be supremum and infinmum densely embedded is isomorphic to the so called MacNeille completion of $P$ (or completion by cuts). It is known that the MacNeille completion of an OML is an ortholattice (see $[7, \mathrm{p} .256])$. If $L$ is an atomic OML then the previous observations imply that its MacNeille completion $\tilde{L}$ is atomic ortholattice and $\tilde{L}$ and $\varphi(L)$ have the same set of all atoms $(\varphi: L \rightarrow \widetilde{L}$ is the embedding). Moreover the fact that $\varphi(L)$ is supremum and infimum dense in $\widetilde{L}$ implies that $\widetilde{L}$ is atomistic and thus the MacNeille completion of an atomic OML is an atomistic ortholattice with the same set of all atoms as $\varphi(L)$.

THEOREM 3.3. Let $L$ be a strongly compactly atomistic OML. Then

(i) $\left(L, \tau_{0}\right)$ is a topological $O M L$.

(ii) The MacNeille completion $\tilde{L}$ of $L$ is an order topological OML.

(iii) $x_{\alpha} \stackrel{\tau_{0}}{\longrightarrow} x$ (in $L$ ) if and only if $x_{\alpha} \stackrel{(0)}{\longrightarrow} x$ (in $\widetilde{L}$ ) for any $x_{\alpha}, x \in L$.

Proof: (ii) Suppose $L$ is a strongly compactly atomistic OML. The MacNeille completion $\widetilde{L}$ of $L$ is a complete ortholattice in which $L$ (we identify $L$ with $\varphi(L)$, where $\varphi: L \rightarrow \widetilde{L}$ is an embedding) is join-dense and meet-dense. The fact that $L$ is join-dense in $\widetilde{L}$ implies that $\widetilde{L}$ is atomistic and that $L$ and $\widetilde{L}$ have the same set of all atoms. Thus $\tilde{L}$ is strongly compactly atomistic and, by Lemma 2.2 and Theorem 3.1, $\widetilde{L}$ is order-topological. We shall show that $\widetilde{L}$ is orthomodular.

Suppose that $x, y \in L, x \leqslant y$ and denote $A_{x}=\{a \in L \mid a \leqslant x$ is an atom $\}$, $A_{y}=\{a \in L \mid a \leqslant y$ is an atom $\}$. Then $A_{x} \subset A_{y}$. Let $\mathcal{E}=\left\{\alpha \subset A_{y} \mid \alpha\right.$ is a finite set and $\left.\alpha \cap A_{x} \neq \emptyset\right\}$. Clearly $\mathcal{E}$ is directed by set inclusion. For any $\alpha \in \mathcal{E}$ we denote $x_{\alpha}=\bigvee \alpha \cap A_{x}, y_{\alpha}=\bigvee \alpha$. Then $x_{\alpha}, y_{\alpha} \in L, x_{\alpha} \leqslant y_{\alpha}$ and $y_{\alpha}=x_{\alpha} \wedge\left(x_{\alpha}^{\perp} \vee y_{\alpha}\right), \alpha \in$ $\mathcal{E}$. Since $x_{\alpha} \nearrow x, y_{\alpha} \nearrow y$ and (in view of the (o)-continuity of $\left.\widetilde{L}\right) x_{\alpha} \vee\left(x_{\alpha}^{\perp} \wedge y_{\alpha}\right) \stackrel{(0)}{\longrightarrow} x \vee$ $\left(x^{\perp} \wedge y\right)$, we obtain $y=x \vee\left(x^{\perp} \wedge y\right)$. We have proved that $\tilde{L}$ is orthomodular.

(iii) Assume that $\left(x_{\alpha}\right)_{\alpha \in \mathcal{E}} \subset L, x \in L$ and $x_{\alpha} \stackrel{\tau_{0}}{\longrightarrow}$ (in $L$ ). By Lemma 2.2 and Theorem 3.1, using the fact that $L$ and $\tilde{L}$ have the same set of atoms, we obtain

$$
x_{\alpha} \stackrel{\tau_{0}}{\longrightarrow} x \text { (in L) if and only if } x_{\alpha} \stackrel{\tau_{\psi}}{\longrightarrow} x
$$

if and only if $\forall a \in A: f_{a}\left(x_{\alpha}\right) \rightarrow f_{a}(x), f_{a^{\perp}}\left(x_{\alpha}\right) \rightarrow f_{a^{\perp}}(x)$ if and only if $x_{\alpha} \stackrel{(o)}{\longrightarrow} x$ (in $\tilde{L}$ ) where $A=\{a \in L \mid a$ is an atom $\}$. 
(i) This follows immediately from (ii) and (iii).

Corollary 3.4. Let $L$ be an atomic $O M L$. The following are equivalent.

(i) The MacNeille completion $\tilde{L}$ of $L$ is an order-topological $O M L$.

(ii) $L$ is strongly compactly atomistic.

THEOREM 3.5. Let $L$ be an atomic OML. The following are equivalent.

(i) A MacNeille completion $\tilde{L}$ of $L$ is a modular $O M L$.

(ii) $L$ is strongly compactly atomistic and $F=\{x \in L \mid x$ is a finite element $\}$ is a modular sublattice in $L$.

Proof: ((i) $\Rightarrow$ (ii)) This follows immediately from Kaplanski's result in [8], and Theorem 3.1, using the fact that $\widetilde{L}$ is complete and the sets of all atoms in $L$ and $\widetilde{L}$ coincide (we indentify $L$ with $\varphi(L)$, where $\varphi: L \rightarrow \widetilde{L}$ is an embedding).

((ii) $\Rightarrow$ (i)) In view of Theorem 3.3 using the claim (ii), a MacNeille completion $\tilde{L}$ of $L$ is an order topological OML. Suppose $x, y, z \in \widetilde{L}, x \leqslant z$. Put $A=\{a \in L \mid a$ is an atom $\}, A_{x}=\{a \in A \mid a \leqslant x\}, A_{y}=\{a \in A \mid a \leqslant y\}, A_{z}=\{a \in A \mid a \leqslant z\}$ and $\mathcal{E}=\left\{\alpha \subset A_{y} \cup A_{z} \mid \alpha\right.$ is a finite set $\}$. Denote $x_{\alpha}=\bigvee \alpha \cap A_{x}, y_{\alpha}=\bigvee \alpha \cap A_{y}, z_{\alpha}=$ $\bigvee \alpha \cap A_{z}$. Since $x_{\alpha}, y_{\alpha}, z_{\alpha} \in F$ and $x_{\alpha} \leqslant z_{\alpha}$, we obtain $x_{\alpha} \vee\left(y_{\alpha} \wedge z_{\alpha}\right)=\left(x_{\alpha} \vee y_{\alpha}\right) \wedge z_{\alpha}$ for every $\alpha \in \mathcal{E}$. The (o)-continuity of $\tilde{L}$ implies that $x_{\alpha} \vee\left(y_{\alpha} \wedge z_{\alpha}\right) \stackrel{(0)}{\longrightarrow x} \vee(y \wedge z)$ and $\left(x_{\alpha} \vee y_{\alpha}\right) \wedge z_{\alpha} \stackrel{(0)}{\longrightarrow}(x \vee y) \wedge z$ and thus $x \vee(y \wedge z)=(x \vee y) \wedge z$.

\section{Order Topological OMLs in the Language of filters; Counterexample}

There are two different possibilities to define the order convergence and order topology in a poset. The net-theoretical approach was developed by G. Birkhoff, O. Frink and others. The study of order convergence in terms of filters was started by A.J.Ward and continued by D.C. Kent. Papers of Marcel Erné about order convergence in lattices and order-topological lattices give several new results, all formulated in the language of filters (see $[10,11])$. Recall the basic definitions.

Let $L$ be a lattice. For a subset $Y$ of $L$, let $Y^{\downarrow}$ and $Y^{\dagger}$ denote the set of all lower and upper bounds of $Y$, respectively. For any filter $\mathcal{F}$ on $L$ we write

$$
\mathcal{F}^{\downarrow}=\bigcup\left\{F^{\downarrow} \mid F \in \mathcal{F}\right\}, \mathcal{F}^{\uparrow}=\bigcup\left\{F^{\uparrow} \mid F \in \mathcal{F}\right\}
$$

We say that $\mathcal{F}$ order converges to a point $x \in L$ (written $\mathcal{F} \stackrel{(0)}{\longrightarrow} x$ ) if $x=\bigvee \mathcal{F}^{\downarrow}=$ $\wedge \mathcal{F}^{\dagger}$. The order topology $\tau_{0}$ on a lattice $L$ (in language of filters) is the finest topology such that the order convergence of filters implies a topological convergence. In general order convergence is distinct from $\tau_{0}$ convergence. Now order convergence of filters is 
said to be topological if it agrees with convergence in the order topology (all in filters language). One can show that at least in lattices (but not in all posets) both definitions of order topology (in terms of nets and in terms of filters) coincide. However as the next example shows, the fact that the order convergence of filters in an $O M L L$ is topological does not imply that the order convergence of nets in $L$ is topological too. In [10], Proposition 2 stated that:

On a lattice $L$, order convergence (of filters) is topological if and only if for all $x \in L, \mathcal{U}(x)$ has a base of intervals. (Here $\mathcal{U}(x)$ means a neighbourhood filter of $x \in L$ in the order topology $\tau_{0}$.)

This statement fails to be true for convergence of nets; see the next example.

EXAMPLE 4.1. Suppose that $H$ is an uncountable set and that for every $\kappa \in H$ the OML $L_{\kappa}$ is finite. Let us put $\widetilde{L}=\prod_{\kappa \in H} L_{\kappa}\left(\widetilde{L}\right.$ is a direct product of OMLs $L_{\kappa}, \kappa \in H$, that is, with coordinatewise defined ordering and orthocomplementation). Evidently $\widetilde{L}$ is complete atomic and (o)-continuous and hence $\widetilde{L}$ is an order-topological OML (in terms of nets) by Theorem 3.1. Let $A$ denote the set of all atoms in $\tilde{L}$. Evidently for every $a=\left(a_{\kappa}\right)_{\kappa \in H} \in A$ there exists $\kappa_{0} \in H$ such that $a_{\kappa_{0}}$ is an atom in $L_{\kappa_{0}}$ and $a_{\kappa}=0 \in L_{\kappa}$ for every $\kappa \neq \kappa_{0}, \kappa \in H$. For every $\alpha \in H$ we denote $A_{\alpha}=\{a=$ $\left(a_{\kappa}\right)_{\kappa \in H} \in A \mid a_{\alpha}$ is an atom in $L_{\alpha}$ and $a_{\kappa}=0 \in L_{\kappa}$ if $\left.\kappa \neq \alpha\right\}$. Then for every $\kappa_{1}, \kappa_{2} \in H$ and every $a \in A_{\kappa_{1}}, b \in A_{\kappa_{2}}$ we have $a \leqslant b^{\perp}$. Now let us denote $F=\{x \in$ $\widetilde{L} \mid x=0$ or $x$ is a join of a finite set of atoms in $\widetilde{L}\}$ and $L=\{y \in \widetilde{L} \mid$ either $y \in F$ or $\left.y^{\perp} \in F\right\}$. Then $L$ is a sub-OML of $\widetilde{L}$ and since $L$ and $\tilde{L}$ have the same set of all atoms, we conclude that $\tilde{L}$ is a MacNeille completion of $L$. Suppose $\left\{\kappa_{1}, \kappa_{2}, \kappa_{3}, \ldots\right\} \subset H$ and $a_{\kappa_{n}} \in A_{\kappa_{n}}, n=1,2, \ldots$ Then $\left\{a_{\kappa_{n}} \mid n=1,2, \ldots\right\}$ is a sequence of mutually orthogonal atoms in the complete OML $\widetilde{L}$ and hence $a_{\kappa_{n}} \stackrel{(0)}{\longrightarrow} 0$ (in $\widetilde{L}$ ). Using Theorems 3.1 and 3.3 we obtain $a_{\kappa_{n}} \stackrel{\tau_{0}}{\longrightarrow} 0$ (in $L$ ). We shall show that $a_{\kappa_{n}} \stackrel{(0)}{\rightarrow} 0$ (in $L$ ). Assume the contrary: $a_{\kappa_{n}} \stackrel{(0)}{\longrightarrow} 0$ (in $L$ ). Then there exist $u_{n}, v_{n} \in L$, $u_{n} \leqslant a_{\kappa_{n}} \leqslant v_{n}(n=1,2, \ldots)$ and $u_{n} \nearrow 0, v_{n} \searrow 0$. Since for every $n=1,2, \ldots$, $\bigvee_{k=n}^{\infty} a_{\kappa_{k}} \leqslant \bigwedge_{k=n}^{\infty} v_{k}=v_{n}$, we have $a_{\kappa_{k}} \leqslant v_{n}$ for every $k=n, n+1, n+2, \ldots$ and hence $v_{n} \notin F$. Thus $v_{n}^{\perp} \in F, n=1,2, \ldots$ For $n=1,2, \ldots$, let $v_{n}^{\perp}=\left(v_{n, \kappa}^{\perp}\right)_{\kappa \in H}$ and $H_{n}=\left\{\kappa \in H \mid v_{n, \kappa}^{\perp} \neq 0 \in L_{\kappa}\right\}$. Then $\bigcup_{n=1}^{\infty} H_{n}$ is at most countable and hence $H \backslash \bigcup_{n=1}^{\infty} H_{n} \neq 0$. Thus for every $\kappa \in H \backslash \bigcup_{n=1}^{\infty} H_{n}$ and every $n=1,2, \ldots$ we have $v_{n, \kappa}^{\perp}=0 \in L_{\kappa}$. Since $v_{n} \searrow 0 \in \tilde{L}$ implies $v_{n}^{\perp} \nearrow 1 \in \tilde{L}$ we have a contradiction. 
Since $a_{\kappa_{n}} \stackrel{\tau_{0}}{\longrightarrow} 0$ (in $L$ ) and $a_{\kappa_{n}} \stackrel{(o)}{\rightarrow} 0$ (in $L$ ), the order convergence of nets in $L$ is not topological. But the order convergence of filters in $L$ is topological by Proposition 2 in [10] mentioned above. To see that we have to show that for any $x \in L$ the neigbourhood filter $\mathcal{U}(x)$ in $\tau_{0}$ has the base of intervals. Really, $L$ is (o)-continuous and hence, in view of Lemma 2.2, $\tau_{0}=\tau_{\psi}$. Thus, for every $a \in A$, intervals $[a, 1],\left[0, a^{\perp}\right]$ are clopen in $\tau_{0}$. If we come back to the proof of Lemma 2.1 we see that for every $x \in L$ and every $U(x) \in \tau_{0}, x \in U(x)$, there exists an interval $\left[x_{\gamma_{0}}, y_{\gamma_{0}}\right]$ which is a clopen set in $\tau_{\psi}$ (and hence in $\tau_{0}$ ) such that $x \in\left[x_{\gamma_{0}}, y_{\gamma_{0}}\right] \subset U(x)$.

REMARK 4.2: Now we come back to Example 4.1 and let $L$ and $\tilde{L}$ have the same meaning as in the mentioned example. We see that $L$ is a topological OML and $\tilde{L}$ is an order topological OML which is a MacNeille completion of $L$. For the chosen sequence $\left(a_{\kappa_{n}}\right)_{n=1}^{\infty}$ of atoms, $a_{\kappa_{n}} \stackrel{(o)}{\longrightarrow} 0$ (in $\tilde{L}$ ), but $a_{\kappa_{n}} \stackrel{(0)}{\rightarrow} 0$ (in $L$ ). This fact disproves (see also [9]) the statement in Birkhoff's book (also for order-topological OMLs) that the order convergence of nets in the MacNeille completion of a poset $P$ induces the order comvergence of nets in $P$ (see [2, cap.X., par.9]). Hence the statement (iii) in Theorem 3.3 cannot be improved.

\section{REFERENCES}

[1] D.H. Adams, 'The completion by cuts of an orthocomplemented modular lattice', Bull. Austral. Math. Soc. 1 (1969), 279-280.

[2] Birkhoff, G., Lattice Theory (Providence, Rhode Island, 1967).

[3] G. Bruns, R.J. Greechie, J. Harding, and M. Roddy, 'Completions of orthomodular lattices', Order 7 (1990), 67-76.

[4] A. Caászár, General topology (Akadémiai Kiadó, Budapest, 1978).

[5] N. Funayama, 'On the completion by cuts of a distributive lattice', Proc. Imp. Acad. Tokyo 20 (1944).

[6] M.F. Janowitz, 'Indexed orthomodular lattices', Math. Z. 119 (1971), 28-32.

[7] G. Kalmbach, Orthomodular lattices (Academic Press, London, 1983).

[8] J. Kaplansky, 'Any orthocomplemented complete modular lattice is continuous geometry', Ann. of Math. 61 (1955), 524-541.

[9] H. Kirchheimová, 'Some remarks on (o)-convergence', in Proc. of the First Winter School of Measure Theory, pp. 110-113 (Liptovský Ján, 1990).

[10] M. Erné, 'Order-topological lattices', Glasgow Math J. 21 (1980), 57-68.

[11] M. Erné and S. Weck, 'Order convergence in lattices', Rocky Mountain J. Math. 10 (1980), 805-818.

[12] S. Pulmannová and Z. Riečanová, 'Compact topological orthomodular lattices', in Contributions to General Algebra 7, pp. 277-282 (Verlag Hölder-Pichler-Tempsky, Wien, 1991).

[13] Z. Riečanová, 'Topologies in atomic quantum logics', Acta Univ. Carolin.-Math. Phys. 30, 143-148. 
[14] Z. Riečanová, 'Applications of topological methods to the completion of atomic orthomodular lattices', Demonstratio Math. 24 (1991), 331-341.

[15] J. Schmidt, 'Zur Kennzeichnung der Dedekind MacNeilleschen Hulle einer Geordneten Menge', Arch. Math. 7 (1956), 241-249.

[16] Tae Ho Choe and R.J. Greechie, 'Representation of locally compact orthomodular lattices', (preprint).

[17] Tae Ho Choe and R.J. Greechie, 'Profinite orthomodular lattices', (preprint).

\section{Department of Mathematics \\ Electrotechnical Faculty of the Slovak Technical University}

Ilkovičova 3

CS-812 19 Bratislava

Czechoslovakia 